Donnelly Centre, University of Toronto

\title{
Disease 'interactome' maps that may one day point to new diagnostics
}

MoGen faculty member Andrew Emili uses powerful mass spectrometers to peer deep into the hidden inner workings of human cells, looking for signatures of disease. To combat the scourge of disease, he believes that we need to develop more effective diagnostic and prognostic tools, which depends in turn on the discovery of more informative "markers" of early stage pathology before irreversible tissue damage is accrued. That's one major reason why his group develops and uses proteomic methods to probe the protein makeup of healthy and diseased human cells in detail.

Every cell in the human body expresses a unique array of proteins - molecules that physically associate with each other in a dynamic and cell type-specific manner to perform intricate biochemical functions - that allow cells to do what they are meant to do. For example, interconnections between certain proteins in cardiac muscle cell are critical for the heart to beat in a regular manner. In skin cells, a different repertoire of proteins interact to detect and repair chromosomal DNA damage following exposure to ultra violet radiation and other genotoxins. In the liver, another set of proteins come together to metabolise stored energy supplies in response to blood borne signaling cues.

Given this intricacy, it should come as no surprise then that human disease can often be traced back to the failure of such proteins to interact in the way they were meant to. Along this line of reasoning, it stands to reason that identifying which are proteins and connections are normally present, and how these go awry in clinical conditions, is likely essential to alleviate or, one day, even cure disease.

But here's the rub: while researchers in MoGen and other laboratories affiliated with the UofT are making progress in understanding which gene products are mutated or otherwise perturbed in diseases like cancer, neurodegeneration and cardiovascular disorders, we still don't have a clue as to how this ultimately impacts human cellular networks. That's because most molecular interaction studies have been restricted to simple cell model systems, primarily because of a lack of suitable tools to probe more complex clinical samples in a native pathophysiological context. This means that clinicians and researchers alike remain in the dark about when faulty associations arise in a particular disorder, or how to detect and treat such anomalies. A useful analogy is to think of the problem as being presented with a box of parts for a new IKEA furniture set that is missing the assembly instructions - you see some bolts, screws and holes, and intuitively know there is a basic relationship between the components, but its not clear what goes where (failure to solve this properly can have catastrophic consequences).

What is urgently needed then is an experimental approach for systematically mapping cellular circuits in patient-derived specimens. One of my lab's goals, therefore, is to create and deploy innovative new methods to reveal normal and diseased protein interaction networks so we can spot the faulty links critical to patient outcomes. These protein maps can also be used to find both signatures of early stage pathology, and mechanistic indicators of how advanced a particular disorder is. The most informative biomarkers hold great promise for diagnosing diseases much earlier than we currently do now, ideally even before any tissue damage appears, which means a physician will be able to prescribe treatments to nip a condition in the bud before it progresses irrevocably to late stage disease, improving outcomes for the patient while reducing the burden on our health care system.

How do we achieve this? Over a decade ago, my lab started building global protein interaction maps for simple models, most notably microbes like yeast and E.coli. Despite gene conservation, there are of course major differences compared to humans, limiting the value of such networks for clinical inference. While a given protein may interact with three other proteins in yeast, its ortholog may have four or more binding partners in humans. So while knowledge of a model organism's protein networks can give clues, one really needs to study patient speci- 


\section{HealTh SCIENCE Inquiry}

mens directly to discover the most clinically relevant associations or perturbations. For various ethical and practical reasons, investigating the inner workings of human tissue directly remains challenging, but my group is generating protein maps for healthy and diseased human cells using our newly developed techniques. Our platform depends on traditional approaches, like biochemical fractionation, in combination with modern cutting edge technologies, like mass spectrometry, to detect the physical connections of thousands of human protein assemblies. Once defined, individual complexes linked to disease progression can be rapidly measured across an array of clinical samples to establish correlation and, potentially causality, with patient outcomes.

Our major focus currently is the networks involved in neurological disease, but we also plan to study connectivity alterations in cancer and heart disease. Our long-term goal is to generate high quality interaction datasets that other biomedical researchers can use to understand the spectrum of altered protein associations that occur in a human clinical setting, and to use these data to devise better tests and drugs to 'correct' such errors. It's a grand vision, and won't happen overnight. Human cell biology is incredibly complicated at the molecular level. Nevertheless, this is why this kind of transformative research is so exciting, and fundamentally so important - it provides essential information to understand how cells work at the most basic level, and is necessary for the development of precision medicine.

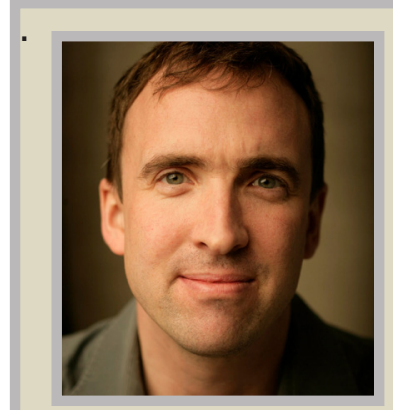

\section{Dr. Andrew Emili}

Dr. Emili is a Pl in the Donnelly Centre at the University of Toronto. He is internationally recognized as a leader in mapping protein interactions and macromolecules on a proteome-scale. Dr. Emili's group develops and applies innovative high-throughput methods to characterize protein networks and complexes of broad biomedical significance, publishing global interaction maps of unprecedented quality, scope and resolution. 